\title{
BMP4 is insufficient to differentiate umbilical cord mesenchymal stem cells into germ cell-like cells in vitro
}

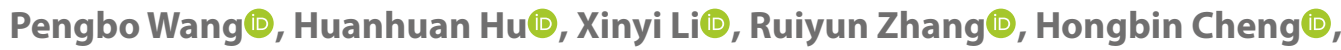 \\ Haixia Qin(1), Guojie Ji(D), Huigen Feng(D), Yanli Liu(D), Juntang Lin(1) \\ Xinxiang Medical University, Xinxiang, China
}

\begin{abstract}
Objectives: Mesenchymal stem cell (MSC)-based therapies are expected to restore the fertility of infertile patients. In addition to MSC-derived paracrine effects to improve reproductive function, the differentiation of MSCs into germ cell (GC)-like cells is still a promising method to repair the injured reproductive system. The aim of this study was to examine the effect and potential mechanism of BMP4 in inducing umbilical cord MSC (UCMSC) transdifferentiation into GC-like cells.

Material and methods: UcMSCs were isolated, cultured and identified by flow cytometry and multilineage differentiation assays. After induction with $12.5 \mathrm{ng} / \mathrm{mL}$ BMP4 for 21 days, UcMSCs were collected for further examination. Immunofluorescence was used to detect the expression of Prdm1 and Prdm14; RT-PCR and RNA sequencing were used to detect differential gene expression (DEGs).

Results: The morphology of UcMSCs became large and flat after treatment with BMP4; the expression of GC-related genes (OCT4, Prdm1, Ifitm3 and Stella) was significantly downregulated, and further immunofluorescence results also confirmed the significant downregulation of Prdm 1 in UcMSCs with BMP4 induction, while the expression of Prdm 14 was significantly upregulated. The results of RNA sequencing and further analysis revealed no explicit correlation between BMP4 induction and the differentiation of UcMSCs into GC-like cells based on the 662 screened DEGs in UcMSCs with or without BMP4 induction.

Conclusions: The differentiation of MSCs into GC-like cells is rather complex, and BMP4 alone is insufficient to induce UcMSCs to differentiate into GC-like cells, regardless of protein level or gene expression level.
\end{abstract}

Key words: germ cell; mesenchymal stem cell; bone morphogenetic protein 4; fertility

Ginekologia Polska 2023; 94, 1: 64-72

\section{INTRODUCTION}

Infertility is one of the diseases that currently plagues humans, and stem cell-based therapies, especially mesenchymal stem cells (MSCs), are expected to restore the fertility of infertile patients. Umbilical cord MSCs (UcMSCs) are widely used in regenerative medicine due to their high pluripotency [1], rich source [2], low immunogenicity and superior immunomodulation capacity [3]. As expected, the therapeutic effects of UcMSC-derived paracrine effects on improving reproductive function and restoring fertility have been confirmed [4]. Several studies have demonstrated that UCMSC transplantation can not only repair premature ovarian failure (POF) caused by chemotherapy by activating the NGF/TrkA pathway but also restore ovarian function by improving the renewal of germline stem cells or activating follicles [5]; simultaneously, UcMSC transplantation can effectively reduce the apoptosis of germ cells in tes- ticular injury caused by hypoglycaemia [6-8]. In addition to MSC-derived paracrine effects on the improvement of reproductive function, the transdifferentiation of MSCs into germ cell (GC)-like cells is still a promising way to repair the injured reproductive system.

Currently, it is well known that ectodermal cells start to secrete bone morphogenetic protein 4 (BMP4) and BMP8 $\beta$ at embryonic stage 5.5, and $\operatorname{Prdm} 1^{+}$cells are considered the precursor cells of primordial germ cells (PGCs). Subsequently, when the secretion of BPM4 and BMP8b reaches the highest level at embryonic stage 6.0 , some Prdm $1^{+}$cells start to differentiate into PGCs. Then, when PGCs migrate to the genital ridge, $\mathrm{PGCs}$ continue to differentiate into GCs [9]. Therefore, based on the above understanding, regents such as BMP4 [10], retinosulfonic acid (RA) [11], all-trans retinoic acid (ATRA) [12], testosterone [13], and follicle stimulating hormone [14] are usually used alone or in combination to

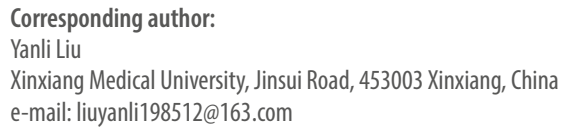


induce the transdifferentiation of MSCs into GC-like cells, which are further identified by cell morphology and the expression of GC-related genes or proteins. As a result, several studies have reported that BMP4 alone and combined with other inducing reagents can indeed promote the gene expression of GC-related genes, such as OCT4, SSEA-1,PRDM1, STELLA, SOX2, C-KIT, PLZF, STRA8, and SCP3, in MSCs in vitro $[15,16]$, and the expression profile of GC-related genes in differentiated MSCs depends not only on the types of MSCs but also on the inducing reagent. Interestingly, all published reports demonstrated that BMP4 and other inducing reagents can upregulate only the expression levels of GC-related genes that were originally expressed in MSCs but have little effect on GC-related genes that were not originally expressed in MSCs.

\section{Objective}

Because MSCs originate from the mesoderm but GCs originate from the ectoderm, we reasonably postulate that the transdifferentiation of MSCs into GC-like cells must be a very complex process, which suggests a sophisticated gene regulation network. Therefore, whether BMP4 alone, which is taken as the core component in inducing the transdifferentiation of MSCs into GC-like cells, can directly drive this transdifferentiation process or is just an enhancer in the transdifferentiation process is still unknown. Consequently, the aim of this study was to examine the promoting effect of BMP4 on the induction of UcMSC transdifferentiation into GC-like cells and preliminarily explore the underlying molecular mechanism, which provides support for the extensive application of UcMSCs in clinical trials.

\section{MATERIAL AND METHODS}

\section{Isolation and culture of UcMSCs}

Programs for sampling human umbilical cord and UcMSCs application were approved by the Ethics Committee of Xinxiang Medical University, all the volunteer donors agreed to use their umbilical cord in this study. Firstly, umbilical cords were obtained from donors at the Third Affiliated Hospital of Xinxiang Medical University; Then, the umbilical cords were stored in ice sterile phosphate-buffered saline (PBS) and delivered to the laboratory; the UcMSCs isolation was finished within eight hours. Subsequently, after removing the arteries and veins in umbilical cord, the Wharton's jelly was transferred to a sterile vial in UltraCULTURE ${ }^{\text {TM }}$ medium (LONZA, USA) and diced into pieces; The explants were transferred to cell culture dish with growth medium (UltraCULTURETM + 2\% Serum replacement (Ultroser GTM, LONZA, USA) $+1 \%$ penicillin and streptomycin mixture). Finally, the cells were cultured undisturbedly until the migration of UcMSCs from the explants at $37^{\circ} \mathrm{C}, 5 \% \mathrm{CO}_{2}$, the fresh medium was changed every other day. When the cells reached
80-90\% confluence (Passage 0, P0), the cells were digested with trypsin (Gibco, USA) and were seeded into new flasks with density of $1.25 \times 10^{4}$ cells $/ \mathrm{cm}^{2}$. The harvested UcMSCs with different passage were conventionally cryopreserved using standard methods in cell freezing medium containing $10 \%$ DMSO for the following experiments.

\section{Flow cytometry}

P3 UcMSCs were used for analysis of cell surface markers, the cell suspensions $\left(1 \times 10^{6}\right.$ cells) were dispersed with $0.125 \%$-trypsin-EDTA and resuspended in PBS supplemented with $0.5 \%$ FBS. Then, the cells were aliquoted into several parts and examined by Human MSC Analysis Kit (562245, BD, USA). Briefly, the cells were respectively incubated with monoclonal antibodies (including CD34/CD45/CD11b/CD19/HLA-DR-PE, CD73-APC, CD90-FITC, and CD105-PerCP-Cy5.5) at $4^{\circ} \mathrm{C}$ in the dark for $20 \mathrm{~min}$, and negative control samples were incubated with associated isotype antibodies. After washing with PBS twice, the cell pellets were re-suspended and analyzed using a Partec CyFlow Cube (German) and the data were processed using FloMax software.

\section{Multipotential differentiation assay}

Adipogenic and osteogenic differentiation of P3 UcMSCs were performed, and the detailed protocols were described in our previous published study [17]. Conventionally, the effect of adipogenic differentiation was evaluated by Oil red $O$ staining, and the effect of osteogenic differentiation was examined by Alizarin red staining. The images were observed and photographed under an inverted microscope (Leica, Germany).

\section{In vitro induction of UcMSCs by BMP4}

The suspended P3 UcMSCs were seeded in a 12-well plate and $75 \mathrm{~cm}^{2}$ flask at a density of $2 \times 10^{3}$ cells $/ \mathrm{cm}^{2}$, respectively. When the cells reached to $50 \%$ confluence, the UcMSCs in experimental group were treated with induction medium (normal growth medium + $12.5 \mathrm{ng} / \mathrm{mL}$ BMP4), and the UcMSCs in the control group were always cultured in normal growth medium. The fresh medium was replaced every three days until the end of the induction period (21 days). Then, the cells seeded in flasks were used to isolate total RNA for the subsequent RT-PCR and RNA-sequence; the cells in plate were used to perform immunofluorescence.

\section{RT-PCR}

The UcMSCs $\left(2 \times 10^{6}\right.$ cells $)$ treated with or without BMP4 were collected for the total RNA isolation using Trizol reagent, and cDNA was harvested from $1 \mu \mathrm{g}$ of total RNA using Primescript RT Master Kit according to the manufacturer's instructions (Takara, Japan). The PCR products 


\begin{tabular}{|c|c|c|c|c|}
\hline Gene & Sequence $\left(5^{\prime}-3^{\prime}\right)$ & $\operatorname{Tm}\left[{ }^{\circ} \mathrm{C}\right]$ & Cycle & Size [bp] \\
\hline \multicolumn{5}{|l|}{ OCT4 } \\
\hline Sense primer & GAGGATCACCCTGGGATATACA & 54.2 & 30 & 550 \\
\hline Antisense primer & GAAAGGGACCGAGGAGTACAGT & 57.2 & 30 & \\
\hline \multicolumn{5}{|l|}{ Ifitm3 } \\
\hline Sense primer & CCAGGAAAAGGAAACTGTTGAG & 53.5 & 30 & 361 \\
\hline Antisense primer & TCCCTAGACTTCACGGAGTAGG & 56.1 & 30 & \\
\hline \multicolumn{5}{|l|}{ Stella } \\
\hline Sense primer & AATCTCCTCCGAGACGTTGATA & 54.5 & 30 & 304 \\
\hline Antisense primer & GGGACATTTGAATGGTCTTGAT & 52.7 & 30 & \\
\hline \multicolumn{5}{|l|}{ PRDM1 } \\
\hline Sense primer & AAGATCAAGTACGAATGCAACG & 52.8 & 30 & 356 \\
\hline Antisense primer & TGCAAGTCTGACATTTGAAAGG & 53.0 & 30 & \\
\hline \multicolumn{5}{|l|}{ GAPDH } \\
\hline Sense primer & ACCACAGTCCATGCCATCAC & 55.9 & 30 & 205 \\
\hline Antisense primer & TCCACCACCCTGTTGCTGTA & 56.5 & 30 & \\
\hline
\end{tabular}

were amplified using $2 \times$ Taq MasterMix (Cwbiotech, China) and visualized by $1.2 \%(\mathrm{w} / \mathrm{v})$ agarose gels with conventional EB staining. The results were normalized against the gray value of the GAPDH band and are represented as targeted mRNA expression relative to that of GAPDH. Primers used are listed in Table 1.

\section{Immunofluorescence}

The cells seeded in plate were conventionally fixed (4\% paraformaldehyde, $15 \mathrm{~min}$ ) and permeabilized (0.05\% Triton X-100, $10 \mathrm{~min}$ ); nonspecific binding was blocked with $1 \%$ BSA (30 min). subsequently, the primary antibodies of anti-Prdm 1 (PA5-20310, rabbit ployclonal antibody, 1:200; invitrogen) and anti-Prdm 14 (PA1-114, rabbit ployclonal antibody, 1:200; invitrogen) were separately added, and the cells were incubated at $4{ }^{\circ} \mathrm{C}$ for $12 \mathrm{~h}$. Thereafter, FITC-conjugated anti-rabbit secondary antibody (1:500; invitrogen) were added and incubated with the cells at $37^{\circ} \mathrm{C}$ for one hour. Cell nuclei was marked with DAPI (Sigma, USA). Finally, the cells were observed and imaged under a fluorescence inverted microscope (Leica, Germany), and the same exposure time was used in all the images for the further quantification of fluorescence value by ImageJ software.

\section{RNA sequencing and analysis}

UcMSCs $\left(2 \times 10^{6}\right)$ with BMP4 induction $(n=3)$ and without BMP4 induction ( $n=3$ ) were collected and delivered to Lc-bio-Technologies (Hangzhou, China) on dry ice. After total RNA isolation and quality confirmation, transcriptome sequencing was performed by Lc-bio-Technologies on the Illumina HiSeq4000 platform using the Illumina paired-end
RNA-seq approach, and a total of million paired-end reads of $2 \times 150 \mathrm{bp}$ (PE150) length were generated. Prior to assembly, the low-quality reads were removed. Subsequently, the high-quality reads between UcMSCs with and without BMP4 induction were aligned to the human reference genome (http://genome.ucsc.edu/) using the TopHat package, and then the reads were mapped to the reference genome. Thirdly, the mapped reads were assembled using StringTie, which was used to further quantify the expression level of mRNAs (genes) by calculating the FPKM. Finally, the differentially expressed genes (DEGs) between UcMSCs with and without BMP4 induction were analysed by the R package-Ballgown with default criteria (fold change $\geq 2$ and statistical significance of $p<0.05$ ), and these DEGs were further analyzed by Gene Ontology (GO) and KEGG database for pathway enrichment. In addition, six typical MSC markers, 14 pluripotent genes, 19 germ cell associated marked genes and 29DNA methylation genes were selected and compared between UcMSCs with and without BMP4 induction.

\section{Statistical analysis}

The data were presented as the mean \pm SD (representative of at least three independent experiments), and Student's t-test was performed to determine statistical significance. $P<0.05$ indicated statistical differences.

\section{RESULTS}

\section{UcMSCs isolation and identification}

As shown in Figure 1A, the spindle-shaped cells started to migrate out from Wharton's jelly fragments after culturing for 5-12 days, and the subcultured UcMSCs exhibited typical 
A

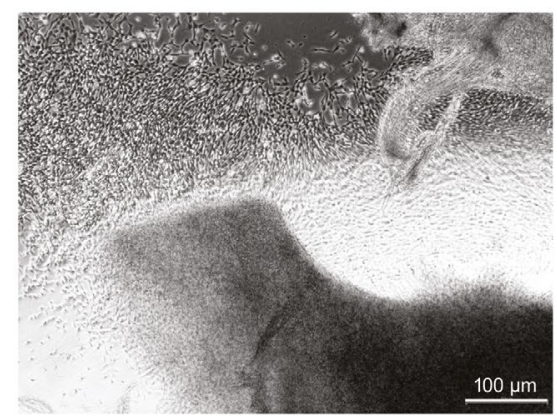

B
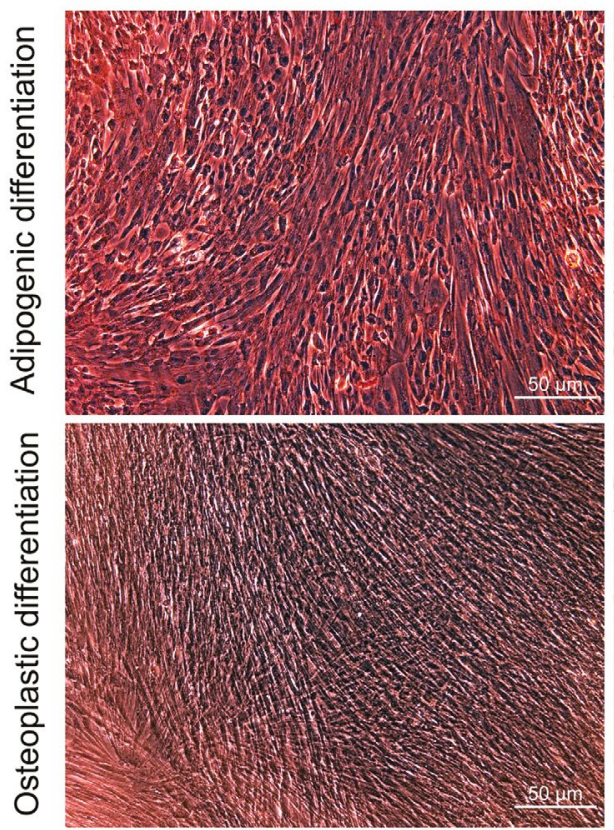

P3 UcMSCs

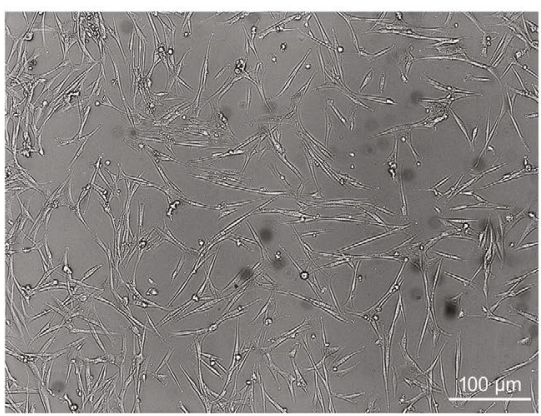

Induction

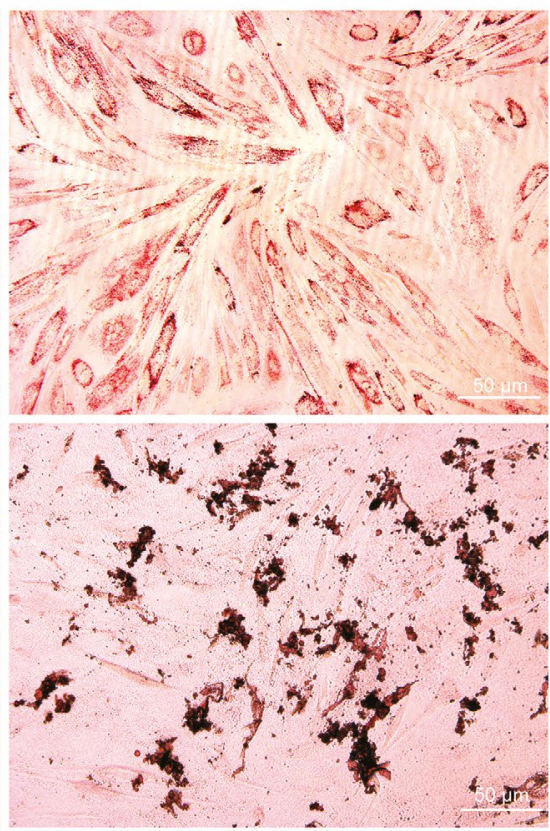

C
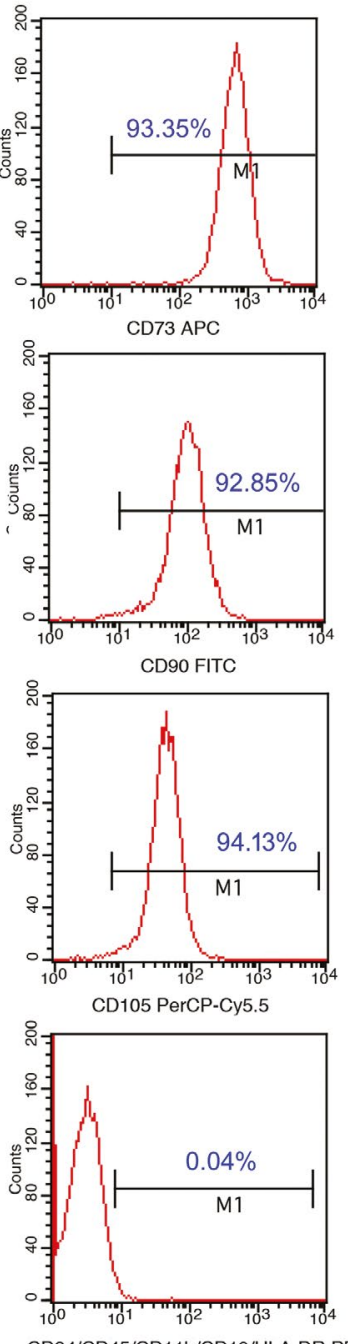

CD34/CD45/CD11b/CD19/HLA-DR PE

Figure 1. The morphology and identification of UcMSCs; A. Representative morphology of primary UcMSCs and passage 3 (P3) UcMSCs; B. Adipogenic and osteogenic differentiation of P3 UcMSCs were performed and the results were respectively examined by positive Oil red $O$ and Alizarin red staining; C. P3 UcMSCs were stained with the corresponding fluorescein-labelled antibodies, and then analyzed by FACS. The results showed that UcMSCs positively expressed typcial MSC markers including CD73, CD90 and CD105, and negatively expressed CD34, CD45, CD11b, CD19 and HLA-DR

characteristics of MSCs (spindle-shaped and fibroblast-like morphology). The subsequent flow cytometry analysis demonstrated that UcMSCs positively expressed typical MSC markers (> 90\%), such as CD73, CD90 and CD105, while negatively expressing CD34/CD45/CD11b/CD19/HLA-DR (Fig. 1B). Finally, as shown in Figure 1C, Furthermore, adipogenic and osteogenic differentiation assays (Fig. 1C) also confirmed the multilineage differentiation potential of UcMSCs.

\section{Effect of BMP4 on differentiation of UcMSCs into GC-like cells}

As shown in Fig 2A, the morphology of UcMSCs with BMP4 induction showed no significant changes in the first three days; starting at day five, the UcMSCs with BMP4 induction exhibited expanded morphology until the end of the induction period, and the volume of UcMSCs increased when compared with the UcMSCs without BMP4 induction. However, UcMSCs without BMP4 induction maintained the typical morphology of MSCs during the induction period. Subsequently, compared to undifferentiated UcMSCs, RT-PCR results demonstrated that the gene expression of GC-related genes (Oct4, Prdm 1, Ifitm3 and Stella) in UcMSCs after BMP4 induction was significantly downregulated ( $p<0.01$, Fig. $2 B$ and $C$ ). In accordance with the RT-PCR results, the following immunofluorescence results also confirmed that the expression of Prdm 1 in UcMSCs with BMP4 induction was significantly downregulated ( $p<0.05$, Fig. $3 A$ and $B$ ), while the expression of Prdm 14 was significantly upregulated $(p<0.05$, Fig. $3 C$ and D). 

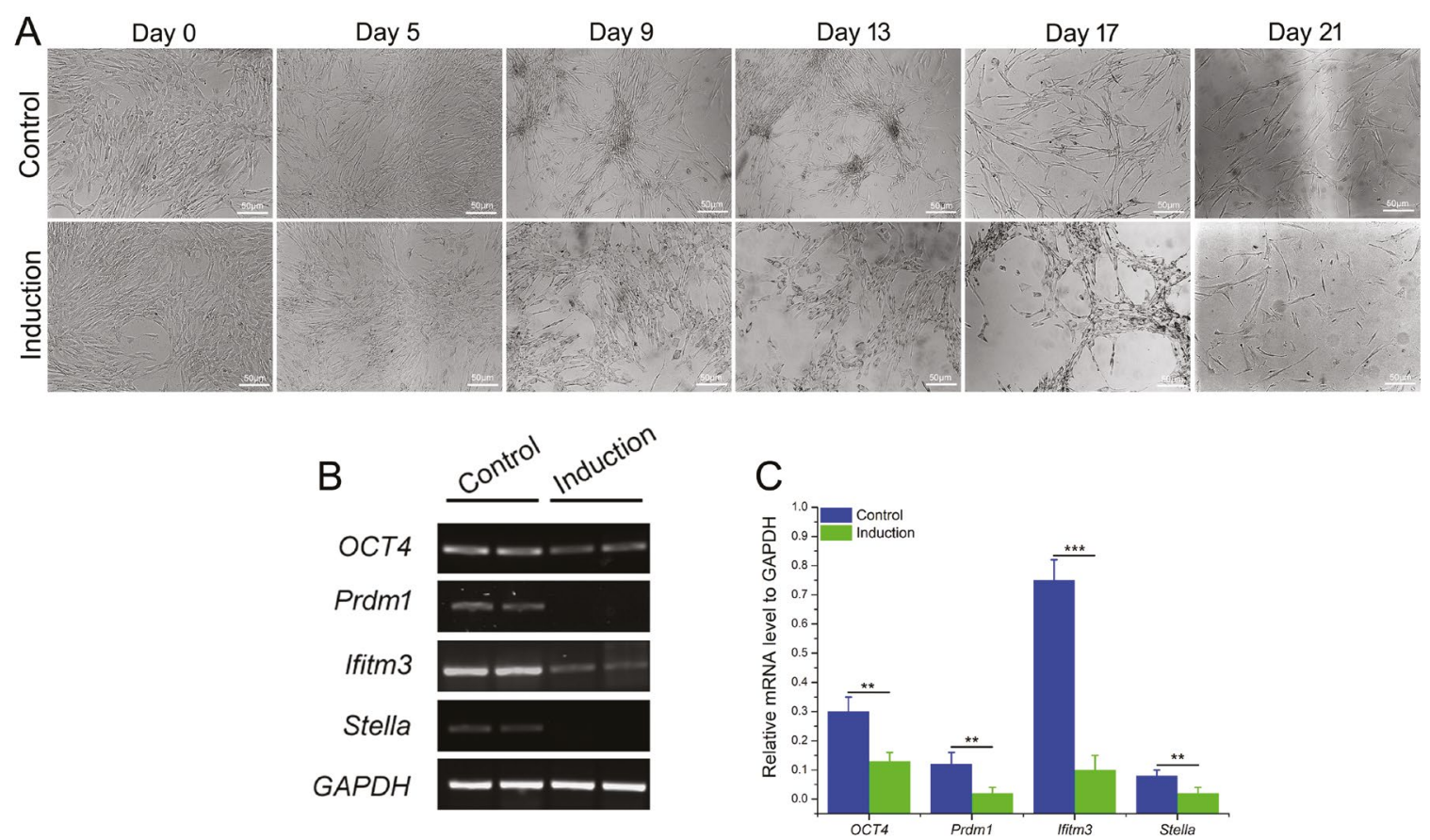

Figure 2. The changes in morphology and gene expression of UCMSCs induced by BMP4; $\mathbf{A}$. Representative morphology of UcMSCs induced by BMP4 at different time points; B. RT-PCR was used to detect the gene expression of GC-related genes (OCT4, Prdm1, Ifitm3, and Stella);

C. Quantitative analysis of germ cell-related genes in UCMSCs. The final results were normalized against the gray value of the GAPDH band and are represented as targeted mRNA expression relative to that of GAPDH. ${ }^{* *} p<0.01 ; * * * 0.001$
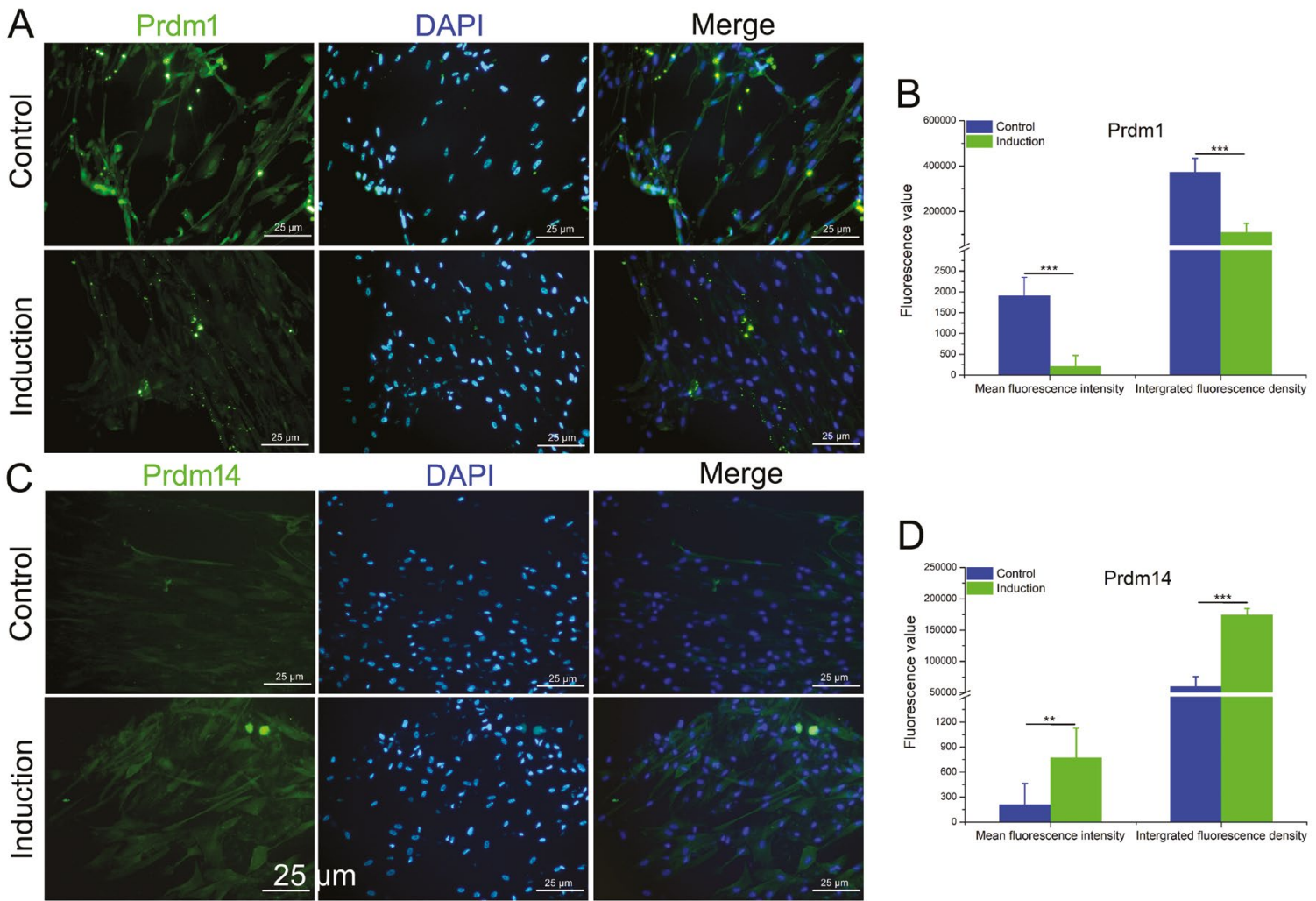

Figure 3. Changes in the typical GC-related proteins in UCMSCs induced by BMP4; A, C. Conventional immunofluorescence was performed to detect the expression of Prdm 1 and Prdm 14 in UcMSCs induced by BMP4; B, D. Expression of Prdm 1 and Prdm 14 in UcMSCs induced by BMP4 was quantified by ImageJ software. ${ }^{* *} \mathrm{p}<0.01,{ }^{* * *} \mathrm{p}<0.001$ 
A

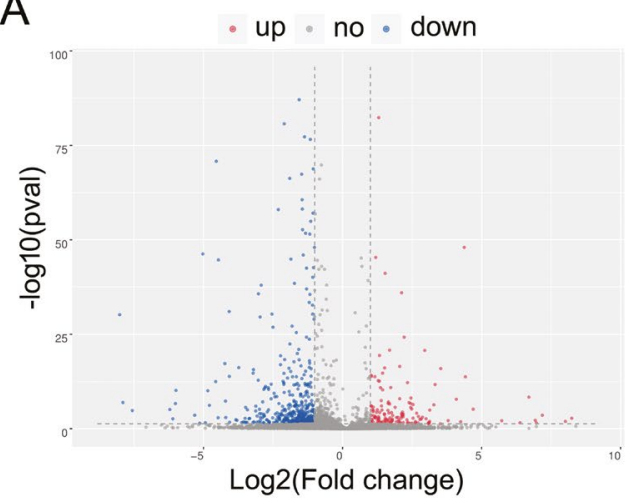

C

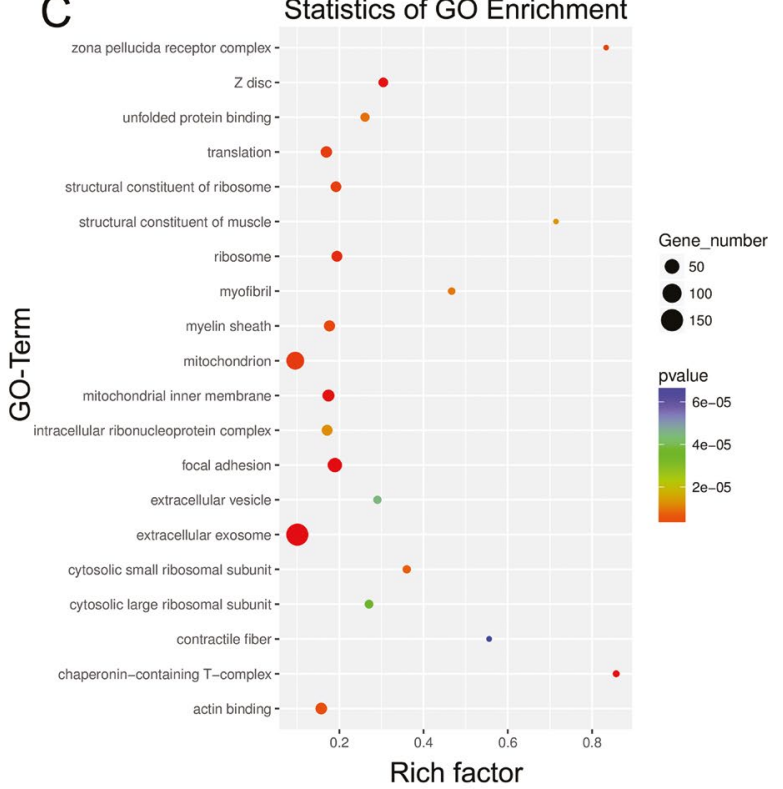

B

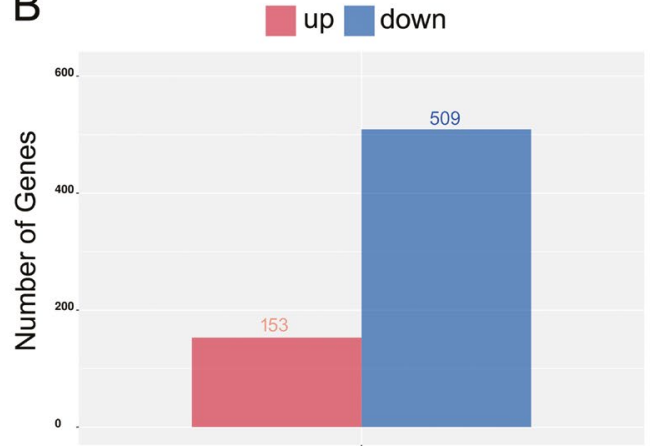

DEGs between naive UcMSCs and induced UcMSCs

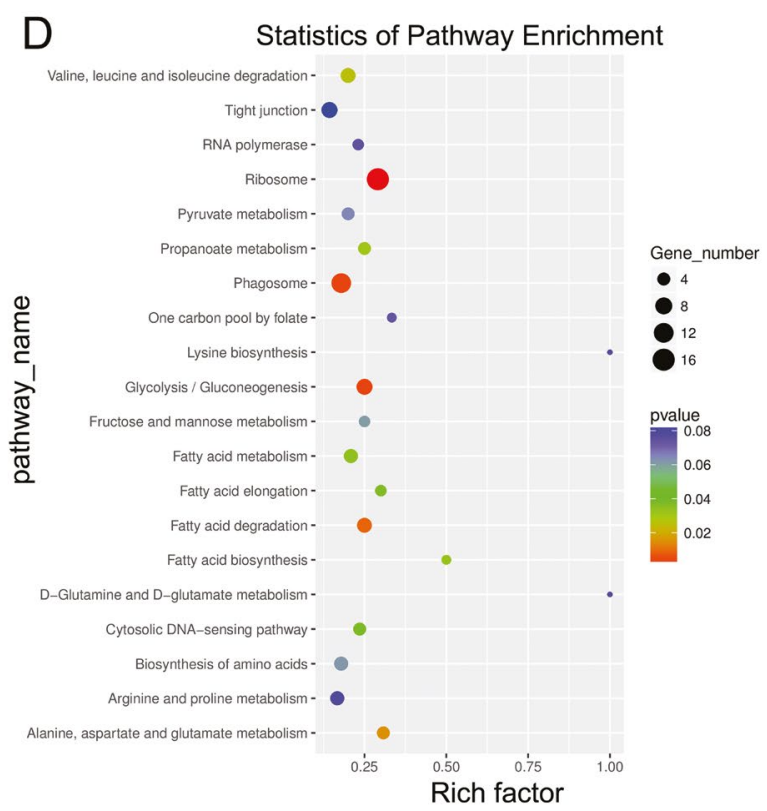

Figure 4. The DEGs between UcMSCs with and without BMP4 induction and the enriched pathways based on these DEGs; A. Volcano map of all genes expressed between UcMSCs with and without BMP4 induction; B. Statistics of DEGs between UcMSCs with and without BMP4 induction. The blue bars indicate downregulated genes, and the pink bars indicate upregulated genes; C. GO term enrichment analysis on DEGs between UcMSCs with and without BMP4 induction; D. KEGG pathway enrichment analysis on DEGs between UcMSCs with and without BMP4 induction

\section{A total of 662 DEGs between UcMSCs with and without BMP4 induction and the pathways enriched with these genes were identified}

RNA-sequences were performed to analyze the DEGs and their derived pathway enrichment in UcMSCs after being induced with BMP4. As shown in Figure $4 A$ and $B$, of the 662 screened DEGs, 153 were upregulated, and the other 509 were downregulated in UcMSCs after induction with BMP4. Subsequently, the screened DEGs were analyzed by $\mathrm{GO}$ annotation database, and the results of $\mathrm{GO}$ enrichment statistics are shown in Figure 4C (top 20 functional classifications), which indicated that most of the DEGs were focused on the functional classifications of extracellular exosomes, mitochondria, focal adhesion and others. Next, the top 20 KEGG pathways enriched with the screened DEGs are shown in Figure 4D, which reveals that most of the DEGs were enriched in the ribosome, phagosome, tight junction, fatty acid metabolism and other pathways. Disappointedly, the above DEGs-enriched functional classifications and pathways exhibit no explicit correlation between BMP4 induction and the differentiation of UcMSCs into GC-like cells. In addition, further analysis is consistent with the above results, and demonstrated that BMP4-induced UcMSCs had nochanges in the expression of typical MSC markers $(p>0.05)$ and had slight decreases in pluripotent, GC-associated and DNA methylated genes but no statistical differences $(p>0.05)$.

\section{DISCUSSION}

MSCs, harvested from bone marrow, umbilical cord, adipose tissue, amniotic membrane and shedding endometrium in menstrual blood have been extensively studied and applied in basic and clinical research due to their rich 
resources, superior proliferative capacity and multiple differentiation potential [2,4-6]. Currently, the application of MSCs, especially UcMSCs, to improve infertility has been extensively studied, and the therapeutic effect has been confirmed in model animals and the clinic $[18,19]$. Ding $L$ et al. [18], showed that UcMSCs combined with collagen scaffolds can restore the ovarian function of long-term infertile patients with POF by activating FOXO3a and FOXO1. Furthermore, UcMSC transplantation could improve chemotherapy-induced ovarian failure, and the NGF/TrkA signaling pathway was involved in the amelioration of POF [5]. Although MSC-derived paracrine effects are suggested to play the main role in promoting the regeneration of the injured reproductive system, the transdifferentiation of MSCs into GC-like cells is still a promising way to improve reproductive function.

Published reports have demonstrated that the cell proliferation, survival and differentiation of PGCs highly depend on the doses of BMP4 secreted by ectoderm cells [20]. As a member of the TGF- $\beta$ superfamily, BMP4 can promote the expression of PGC-specific genes such as Stella, fragilis, and Mvh [16], and BMP4 can induce embryonic stem cells (ESCs) and induced pluripotent stem cells (iPSCs) to differentiate into PGC-like cells [21]. Furthermore, Shirazi et al., [22] and N. Li et al., [15] demonstrated that SSEA-1 ${ }^{+}$MSCs are capable of differentiating into GC-like cells after induction with BMP4 or RA. Additionally, MSCs derived from different sexes or tissues exhibit various differentiation abilities and unique expression profiles of GC-related genes; for example, male-derived BM-MSCs tend to differentiate into male germ cells rather than female germ cells [23], and $12.5 \mathrm{ng} / \mathrm{mL}$ of BMP4 is sufficient to induce canine adipose-derived MSC differentiation into PGC-like cells [10], while for human amniotic membrane derived MSCs and UcMSCs, $25 \mathrm{ng} / \mathrm{mL}$ BMP4 is required to induce differentiation into PGC-like cells [15]. Subsequent treatment with inducing factors, such as RA, can further promote the differentiation of PGC-like cells into GC-like cells [11].

Therefore, the effect of BMP4 on inducing UcMSC differentiation into GC-like cells was systemically examined in this study. Consistent with a published report, the morphology of UcMSCs became large and flat after treatment with BMP4. However, the gene expression of GC-related genes (OCT4, Prdm 1, Ifitm3 and Stella) was significantly downregulated, and the further immunofluorescence results also confirmed the significant down regulation of Prdm 1 in UcMSCs with BMP4 induction, while the expression of Prdm14 was significantly upregulated. Prdm1, also known as B lymphocyte-induced maturation protein-1, is widely expressed during development, including PGCs migration [24]. Existing studies have shown that Prdm $1^{+}$cells are the precursor cells of PGCs during the development of GCs, the loss of Prdm 1 inhibits the migration and proliferation of PGCs in the genital ridge, and the loss of endogenous Prdm 1 in PGCs cannot be compensated by exogenous supplementation with Prdm 1 [24]. Simultaneously, Prdm 1 can synergistically act with Prdm 14 to induce epigenetic reprogramming in PGCs and early GCs, indicating their synergistic effect in the development of the germ cell lineage [25]. Consequently, our results were inconsistent with the published reports [15], and preliminarily demonstrated that BMP4 alone is insufficient to directly induce the differentiation of UcMSCs into GC-like cells, and the decrease in OCT4 gene expression may be caused by cell ageing.

Next, RNA sequencing was performed to identify the transcriptome differences of BMP4-induced UcMSCs. Consistent with our previous findings, the results of $\mathrm{GO}$ annotation and KEGG enrichment analysis based on the 662 screened DEGs in BMP4-induced UcMSCs exhibited no explicit correlation between BMP4 induction and the differentiation of UcMSCs into GC-like cells, and the analysis also showed that the value of FPKM in typical MSC markers and the expression of pluripotent, GC-associated and DNA methylated genes all had no significant change in UcMSCs with or without BMP4 induction (Fig. 5). The above results indicated that BMP4-induced UcMSCs are unable to differentiate into GC-like cells. Additionally, some of the screened DEGs were enriched in pathways of fatty acid metabolism, which can activate the p53 signaling, and in turn cause cell cycle arrest, thereby inhibiting the proliferation of PGCs and leading to apoptosis of PGCs [26].

\section{CONCLUSIONS}

In summary, this study indicated that the transdifferentiation of MSCs into GC-like cells is rather complex, which suggests a sophisticated gene regulation network operating in a spatial and temporal manner, and BMP4 alone is insufficient to induce UcMSCs to differentiate into GC-like cells, regardless of the protein level or gene expression level. Therefore, more attention should be paid to the further selection and optimization of appropriate induction media with specific inducing reagents, which will provide support for the extensive application of UcMSCs in restoring reproductive function. 
A

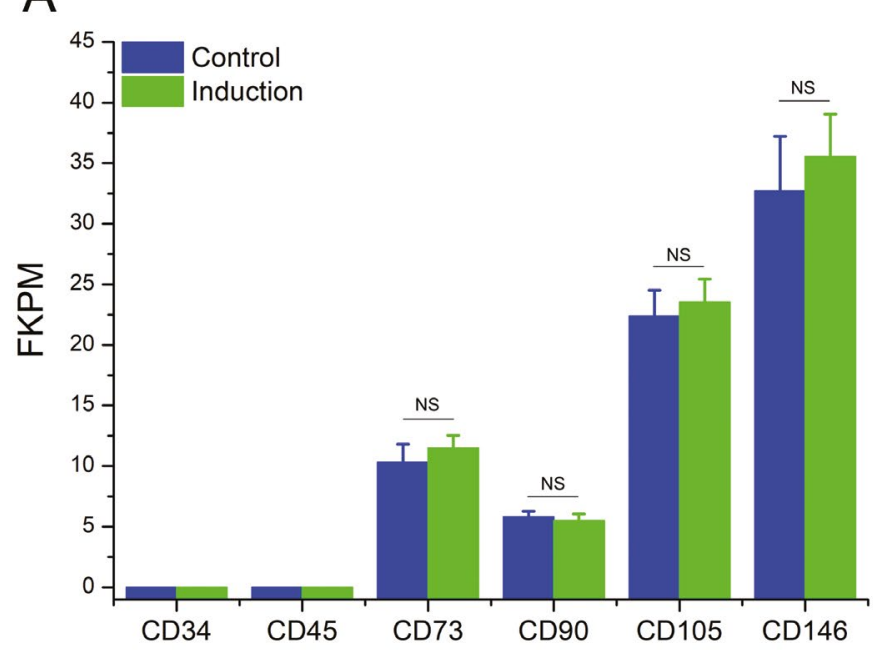

C

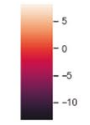

Marker genes of germ cell

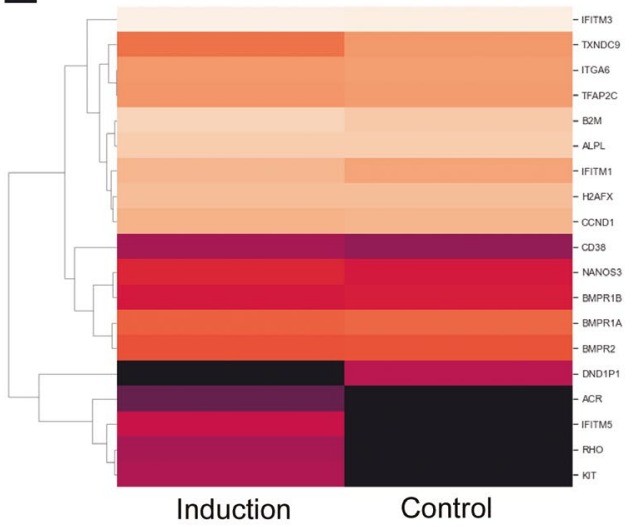

B

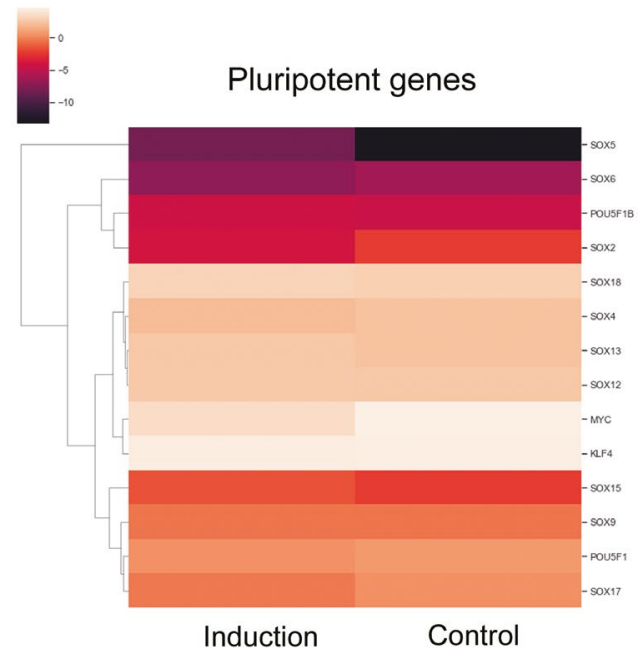

D

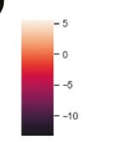

DNA mehtylated genes

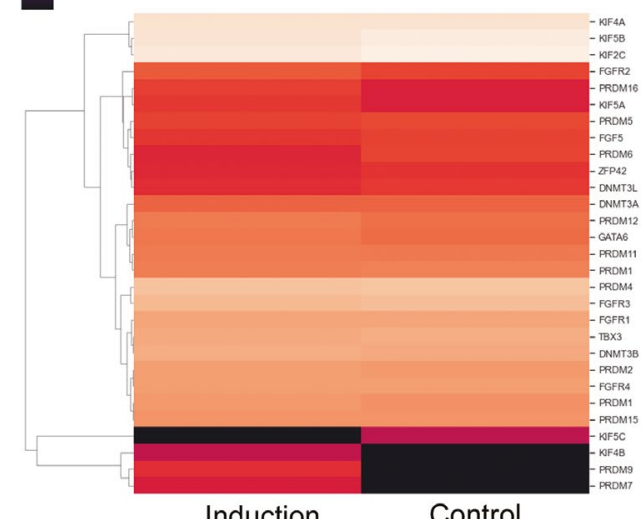

Induction

Control

Figure 5. Further difference analysis of targeted genes; A. Gene expression levels of typical MSC markers in UcMSCs with and without BMP4 induction, and FPKM value represents the gene expression level; B-D. Cluster analysis of pluripotent, GC-associated and DNA methylated gene expression levels in UcMSCs with and without BMP4 induction

\section{Funding}

We would like to acknowledge the Henan Province Foundation (20B310013, 162102210117 and 192102310332) and Xinxiang City Foundation (GG2019022) for the financial support.

\section{Conflict of interest}

All authors declare no conflict of interest regarding the publication of this paper.

\section{REFERENCES}

1. Guo $X$, Tang $Y$, Zhang $P$, et al. Effect of ectopic high expression of transcription factor OCT4 on the "stemness" characteristics of human bone marrow-derived mesenchymal stromal cells. Stem Cell Res Ther. 2019; 10(1): 160, doi: 10.1186/s13287-019-1263-4, indexed in Pubmed: 31159871.
2. Asgari HR, Akbari $M$, Yazdekhasti $H$, et al. Comparison of human amniotic, chorionic, and umbilical cord multipotent mesenchymal stem cells regarding their capacity for differentiation toward female germ cells. Cell Reprogram. 2017; 19(1): 44-53, doi: 10.1089/cell.2016.0035, indexed in Pubmed: 28112985.

3. Samiec M, Opiela J, Lipiński D, et al. Trichostatin A-mediated epigenetic transformation of adult bone marrow-derived mesenchymal stem cells biases the in vitro developmental capability, quality, and pluripotency extent of porcine cloned embryos. Biomed Res Int. 2015; 2015: 814686, doi: 10.1155/2015/814686, indexed in Pubmed: 25866813.

4. Ling Li, Feng $X$, Wei T, et al. Human amnion-derived mesenchymal stem cell (hAD-MSC) transplantation improves ovarian function in rats with premature ovarian insufficiency (POI) at least partly through a paracrine mechanism. Stem Cell Res Ther. 2019; 10(1): 46, doi: 10.1186/s13287019-1136-x, indexed in Pubmed: 30683144.

5. Zheng Q, Fu X, Jiang J, et al. Umbilical cord mesenchymal stem cell transplantation prevents chemotherapy-induced ovarian failure via the NGF/TrkA pathway in rats. Biomed Res Int. 2019; 2019: 6539294, doi: 10.1155/2019/6539294, indexed in Pubmed: 31240219. 
6. Dessouky AA, Gouda ZA, Arafa MAA, et al. Hypoxia-preconditioned human umbilical cord blood-derived mesenchymal stem cells mitigate hypoglycemic testicular injury induced by insulin in rats. Cells Tissues Organs. 2020; 209(2-3): 83-100, doi: 10.1159/000510363, indexed in Pubmed: 33113534.

7. Bochinski D, Lin GT, Nunes $L$, et al. The effect of neural embryonic stem cell therapy in a rat model of cavernosal nerve injury. BJU Int. 2004; 94(6): 904-909, doi: 10.1111/j.1464-410X.2003.05057.x, indexed in Pubmed: 15476533.

8. Qiu X, Villalta J, Ferretti L, et al. Effects of intravenous injection of adipose-derived stem cells in a rat model of radiation therapy-induced erectile dysfunction. J Sex Med. 2012; 9(7): 1834-1841, doi: 10.1111/j.1 743-6109.2012.02753.x, indexed in Pubmed: 22548750.

9. Ohinata $\mathrm{Y}, \mathrm{Ohta} \mathrm{H}$, Shigeta $\mathrm{M}$, et al. A signaling principle for the specification of the germ cell lineage in mice. Cell. 2009; 137(3): 571-584, doi: 10.1016/j.cell.2009.03.014, indexed in Pubmed: 19410550.

10. Wei Y, Fang J, Cai S, et al. Primordial germ cell-like cells derived from canine adipose mesenchymal stem cells. Cell Prolif. 2016; 49(4):503-511, doi: 10.1111/cpr.12271, indexed in Pubmed: 27374854.

11. Ghorbanlou M, Abdanipour A, Shirazi R, et al. Indirect co-culture of testicular cells with bone marrow mesenchymal stem cells leads to male germ cell-specific gene expressions. Cell J. 2019; 20(4): 505-512, doi: 10.22074/cellj.2019.5654, indexed in Pubmed: 30123996.

12. Kashyap V, Rezende NC, Scotland KB, et al. Regulation of stem cell pluripotency and differentiation involves a mutual regulatory circuit of the NANOG, OCT4, and SOX2 pluripotency transcription factors with polycomb repressive complexes and stem cell microRNAs. Stem Cells Dev. 2009; 18(7): 1093-1108, doi: 10.1089/scd.2009.0113, indexed in Pubmed: 19480567.

13. Huang $P$, Lin LiM, Wu XY, et al. Differentiation of human umbilical cord Wharton's jelly-derived mesenchymal stem cells into germ-like cells in vitro. J Cell Biochem. 2010; 109(4): 747-754, doi: 10.1002/jcb.22453, indexed in Pubmed: 20052672

14. Lee $\mathrm{YM}$, Kim TH, Lee JH, et al. Overexpression of Oct4 in porcine ovarian stem/stromal cells enhances differentiation of oocyte-like cells in vitro and ovarian follicular formation in vivo. J Ovarian Res. 2016; 9: 24, doi: 10.1186/s13048-016-0233-z, indexed in Pubmed: 27067537.

15. Li N, Pan S, Zhu H, et al. BMP4 promotes SSEA-1(+) hUC-MSC differentiation into male germ-like cells in vitro. Cell Prolif. 2014; 47(4): 299-309, doi: 10.1111/cpr.12115, indexed in Pubmed: 24923741.

16. Mazaheri Z, Movahedin M, Rahbarizadeh F, et al. Different doses of bone morphogenetic protein 4 promote the expression of early germ cell-specific gene in bone marrow mesenchymal stem cells. In Vitro Cell Dev Biol Anim. 2011; 47(8): 521-525, doi: 10.1007/s11626-011-9429-0, indexed in Pubmed: 21717271.

17. Liu Y, Niu R, Li W, et al. Biological characteristics of human menstrual blood-derived endometrial stem cells. J Cell Mol Med. 2018; 22(3): 1627-1639, doi: 10.1111/jcmm.13437, indexed in Pubmed: 29278305.

18. Ding L, Yan G, Wang B, et al. Transplantation of UC-MSCs on collagen scaffold activates follicles in dormant ovaries of POF patients with long history of infertility. Sci China Life Sci. 2018; 61(12): 1554-1565, doi: 10.1007/s11427-017-9272-2, indexed in Pubmed: 29546669.

19. Lai $D$, Wang $F$, Yao $X$, et al. Human endometrial mesenchymal stem cells restore ovarian function through improving the renewal of germline stem cells in a mouse model of premature ovarian failure. J Transl Med. 2015; 13: 155, doi: 10.1186/s12967-015-0516-y, indexed in Pubmed: 25964118.

20. Mochizuki K, Tando Y, Sekinaka T, et al. SETDB1 is essential for mouse primordial germ cell fate determination by ensuring BMP signaling. Development. 2018; 145(23), doi: 10.1242/dev.164160, indexed in Pubmed: 30446626.

21. Murakami K, Günesdogan U, Zylicz JJ, et al. NANOG alone induces germ cells in primed epiblast in vitro by activation of enhancers. Nature. 2016; 529(7586): 403-407, doi: 10.1038/nature16480, indexed in Pubmed: 26751055.

22. Shirazi R, Zarnani AH, Soleimani M, et al. Differentiation of bone marrow-derived stage-specific embryonic antigen 1 positive pluripotent stem cells into male germ cells. Microsc Res Tech. 2017; 80(4): 430-440, doi: 10.1002/jemt.22812, indexed in Pubmed: 27990704.

23. Katsara O, Mahaira LG, Iliopoulou EG, et al. Effects of donor age, gender, and in vitro cellular aging on the phenotypic, functional, and molecular characteristics of mouse bone marrow-derived mesenchymal stem cells. Stem Cells Dev. 2011; 20(9): 1549-1561, doi: 10.1089/scd.2010.0280, indexed in Pubmed: 21204633.

24. Ohinata Y, Payer B, O'Carroll D, et al. Blimp1 is a critical determinant of the germ cell lineage in mice. Nature. 2005; 436(7048): 207-213, doi: 10.1038/nature03813, indexed in Pubmed: 15937476.

25. Bao S, Leitch HG, Gillich A, et al. The germ cell determinant Blimp1 is not required for derivation of pluripotent stem cells. Cell Stem Cell. 2012; 11(1): 110-117, doi: 10.1016/j.stem.2012.02.023, indexed in Pubmed: 22770244.

26. Wagner W, Horn P, Castoldi M, et al. Replicative senescence of mesenchymal stem cells: a continuous and organized process. PLoS One. 2008; 3(5): e2213, doi: 10.1371/journal.pone.0002213, indexed in Pubmed: 18493317. 